\title{
LOCAIS E PERÍODO DE ALIMENTAÇÃO DA CIGARRINHA VETORA DE Xylella fastidiosa, Bucephalogonia xanthophis (BERG) (HEMIPTERA: CICADELLIDAE), EM MUDAS CÍTRICAS ${ }^{1}$
}

\author{
MARCELO PEDREIRA DE MIRANDA ${ }^{2}$, DENISENUNES VIOLA ${ }^{3}$ RODRIGO NEVES MARQUES ${ }^{4}$ \\ JEAN PATRICK BONANI ${ }^{5}$, JOÃO ROBERTO SPOTTI LOPES ${ }^{6}$
}

RESUMO--A cigarrinha Bucephalogonia xanthophis (Berg) (Hemiptera: Cicadellidae) é um importante vetor da bactéria Xylella fastidiosa, agente causal da clorose variegada dos citros. Este trabalho teve como objetivo identificar o local preferido de alimentação e o período de maior atividade alimentar desta cigarrinha em citros, no sentido de elucidar o comportamento alimentar relacionado à transmissão da bactéria. O local de alimentação foi estudado em ensaio de escolha, no qual 30 insetos adultos foram liberados em gaiolas de observação $(\mathrm{n}=10)$ contendo uma muda de laranja-doce [Citrus sinensis (L.) Osbeck]. Após 1; 15; 21; 25; $39 ; 45$ e 49 h da liberação, contaram-se os insetos na parte superior (ramos com brotações) e inferior (haste principal, até H" $40 \mathrm{~cm}$ de altura) da muda. Nos ramos da parte superior, avaliou-se a preferência entre a haste, o pecíolo e o limbo foliar. Em um segundo ensaio, 20 machos e 20 fêmeas de B. xanthophis foram confinados individualmente sobre a haste de 'seedlings' de laranja-doce para determinar os períodos de alimentação, quantificando-se a excreção de 'honeydew' (medida indireta da ingestão) em períodos sucessivos de dia e noite, durante $48 \mathrm{~h}$. A maioria dos indivíduos de B. xanthophis preferiu a haste dos ramos novos (62\%), na parte superior da muda cítrica $(91 \%)$. Nos 'seedlings', observou-se maior volume de excreção e proporção de indivíduos excretando durante a fotofase, independentemente do sexo. Portanto, em estudos de transmissão de X. fastidiosa, deve-se considerar a preferência de B. xanthophis pela haste de brotações cítricas e sua maior atividade alimentar durante a fotofase.

Termos para indexação: Cicadellinae, Comportamento alimentar, Clorose variegada dos citros.

\section{FEEDING SITES AND FOOD INTAKE OF Bucephalogonia xanthophis (BERG) (HEMIPTERA: CICADELLIDAE), A SHARPSHOOTER VECTOR OF Xylella fastidiosa, ON CITRUS PLANTS}

\begin{abstract}
The sharpshooter Bucephalogonia xanthophis (Berg) (Hemiptera: Cicadellidae) is an important vector of Xylella fastidiosa, a bacterium that causes citrus variegated chlorosis. This study aimed to identify the preferred feeding sites and periods of this vector on citrus, in order to improve our understanding of the feeding behavior related to the transmission of this pathogen. The feeding sites were determined in a choice experiment, in which 30 adults were released inside observation chambers $(\mathrm{n}=10)$ containing a sweet orange seedling [Citrus sinensis (L.) Osbeck]. One, fifteen, twenty-one, twenty-five, thirty-nine, forty-five, and forty-nine hours after the release, the number of insects on the upper (stem with sprouts) and bottom (primary stem, up to $40 \mathrm{~cm}$ above soil) parts of the plant was recorded. To determine the feeding periods, 20 males and 20 females of B. xanthophis were individually confined on the stems of sweet orange seedlings, and the honeydew excretion (indirect measure of ingestion) was quantified for 48 hours, during periods of day and night. Most B. xanthophis individuals (91\%) were observed on the upper part of the plant, mainly on the stems of the young shoots $(62 \%)$. A larger volume of excretion and higher proportion of excreting individuals were observed during the photophase for both males and females, indicating that daytime is the preferred feeding period for this vector. Therefore, in transmission studies of $X$. fastidiosa one should consider the preference of B. xanthophis for stems of young shoots and its higher feeding activity during the photophase.
\end{abstract}

Index terms: Cicadellinae, Feeding behavior, Citrus variegated chlorosis.

${ }^{1}$ (Trabalho 035-08). Recebido em: 31-01-2008. Aceito para publicação em: 13-08-2008.

${ }^{2}$ Eng. Agr., Dr. em Entomologia, Departamento de Entomologia, Fitopatologia e Zoologia Agrícola - ESALQ/USP. Av. Pádua Dias, 11, CP 9, CEP: 13.418-900 - Piracicaba-SP - Brasil. mmiranda@esalq.usp.br

${ }^{3}$ Estat., Dr., Professora do Departamento de Estatística - UFBA, Av. Adhemar de Barros, s/n - Campus de Ondina - CEP: 40.170-110 - Salvador-BA Brasil.viola@ufba.br

${ }^{4}$ Eng. Agr., MS. em Entomologia, Departamento de Entomologia, Fitopatologia e Zoologia Agrícola - ESALQ/USP. Av. Pádua Dias, 11, CP 9, CEP: 13.418-900 - Piracicaba-SP. rnmarque@esalq.usp.br

${ }^{5}$ Eng. Agr., MS. em Entomologia, Departamento de Entomologia, Fitopatologia e Zoologia Agrícola - ESALQ/USP. Av. Pádua Dias, 11, CP 9, CEP: 13.418-900 - Piracicaba-SP. jpbonani@esalq.usp.br

${ }^{6}$ Eng. Agr., Dr., Professor do Entomologia, Departamento de Entomologia, Fitopatologia e Zoologia Agrícola - ESALQ/USP. Av. Pádua Dias, 11, CP 9, CEP: 13.418-900 - Piracicaba-SP. jlopes@esalq.usp.br 


\section{INTRODUÇÃO}

Xylella fastidiosa é uma bactéria que se multiplica no xilema de plantas, causando doenças em diversas culturas de importância econômica (Purcell \& Hopkins, 1996). No Brasil, é agente causal da Clorose Variegada dos Citros (CVC), doença que afeta laranja-doce [Citrus sinensis (L.) Osbeck] e que se caracteriza por sintomas de clorose na face superior das folhas e drástica redução no tamanho dos frutos (Rossetti et al., 1997; Lee et al., 1993). Esse patógeno é transmitido por cigarrinhas das famílias Cicadellidae (subfamília Cicadellinae) e Cercopidae, que se alimentam no xilema das plantas (Purcell, 1989). Em citros, já foram identificadas várias espécies de cigarrinhas vetoras, sendo todas representantes de Cicadellinae (Roberto et al., 1996; Krügner et al., 2000; Descobertos, 1999; Yamamoto et al., 2002; Yamamoto et al., 2007).

A disseminação da CVC nos pomares ocorre pela ação dos insetos vetores a partir de mudas ou árvores cítricas infectadas (Lopes, 1999). Dentre as cigarrinhas identificadas como vetoras, Bucephalogonia xanthophis (Berg) (Hemiptera: Cicadellidae) destaca-se por ser freqüentemente observada em viveiros abertos (Roberto et al., 2000) e pomares cítricos em formação (Yamamoto et al., 2001).

Em citros, as cigarrinhas vetoras de $X$. fastidiosa apresentam uma baixa eficiência de transmissão quando comparadas aos vetores da estirpe de videira (Lopes, 1999). Esse fato pode estar relacionado com uma baixa concentração da bactéria em citros (Almeida et al., 2001) e uma distribuição irregular de X. fastidiosa nas plantas hospedeiras (Mizubuti et al., 1994), o que pode reduzir a eficiência de aquisição pelas cigarrinhas. Além desses fatores, pode ocorrer uma baixa taxa de sobrevivência de infecções iniciais de X. fastidiosa em citros, após sua inoculação por vetores (Lopes, 1999).

Aspectos relacionados à interação inseto-planta também podem influenciar na eficiência de transmissão. O local de alimentação na planta (Lopes, 1996), a baixa taxa de ingestão observada em citros em relação a outras plantas hospedeiras (Milanez et al., 2003) e as condições hídricas do solo que influenciam na sobrevivência e alimentação da cigarrinha na planta hospedeira (Pereira et al., 2005), possivelmente, afetam a eficiência com que diferentes espécies de cigarrinhas adquirem ou inoculam $X$. fastidiosa.

Como a distribuição de $X$. fastidiosa é desuniforme na planta cítrica (Queiroz-Voltan \& Paradela Filho, 1999), o local de alimentação do vetor é um dos principais fatores que podem influenciar na probabilidade de aquisição ou inoculação do patógeno. Cada espécie vetora tem preferência por determinada parte do ramo cítrico (haste ou folha), podendo escolher entre ramos novos ou mais desenvolvidos (Gravena et al., 1997). Para Dilobopterus costalimai Young, o local de preferência e a atividade alimentar podem variar de acordo com o período do dia (Marucci et al., 2004; Montesino et al., 2006).

Conhecer as características do comportamento alimentar de cigarrinhas vetoras de $X$. fastidiosa em citros é importante para melhor compreender o processo de transmissão desse patógeno e definir táticas de controle mais eficientes que possam ser implementadas no manejo da CVC. Este trabalho teve como objetivo determinar o local preferido de alimentação e o período de maior atividade alimentar de B. xanthophis em mudas cítricas.

\section{MATERIAL E MÉTODOS}

\section{Obtenção de cigarrinhas e plantas-teste}

A cigarrinha $B$. xanthophis foi criada em plantas de Vernonia condensata Baker (Asteraceae), conforme descrito por Marucci et al. (2003), de modo a obter adultos com idade padronizada. Para o estudo de seleção do local de alimentação, usaram-se mudas com 1 ano de idade de laranja-doce [Citrus sinensis (L.) Osbeck], variedade Pêra, enxertada sobre citrumelo Swingle [Citrus paradisi Macf. x Poncirus trifoliata (L.) Raf.]. No estudo de horário de alimentação, utilizaram-se 'seedlings' de laranja-doce, variedade Pêra, obtidas a partir de sementes. Os dois tipos de plantas foram produzidas em viveiros telados, em vasos de $4 \mathrm{~L}$ com substrato comercial Plantmax ${ }^{\circledR}$, sendo adubadas periodicamente com o fertilizante de liberação lenta, fórmula 2204-08 (Osmocote ${ }^{\circledR}$ ).

\section{Seleção do local de alimentação na planta cítrica}

Em um experimento de livre escolha, avaliou-se a preferência da cigarrinha por duas partes principais da muda cítrica: a) superior (ou copa), formada por três ramos secundários (brotações) com cerca de $20 \mathrm{~cm}$ cada, e b) inferior, constituída pela haste principal (enxerto + porta-enxerto) com, aproximadamente, $40 \mathrm{~cm}$ de altura. Nos ramos da parte superior da muda, avaliou-se a preferência entre a haste, pecíolos e limbos foliares. Utilizaram-se gaiolas de observação (base de $63 \times 63 \mathrm{~cm}$ e $120 \mathrm{~cm}$ de altura) com estrutura de madeira e revestidas com acrílico transparente e tela antiafídica, descritas por Marucci et al. (2004). O experimento foi composto por 10 repetições, sendo cada uma constituída por uma gaiola de observação contendo uma muda cítrica, no interior da qual foram liberadas 30 cigarrinhas adultas (machos e fêmeas em proporções semelhantes) com 7-14 dias de idade. A liberação dos insetos foi feita no final da tarde $(17 \mathrm{~h})$ e as avaliações foram realizadas após $1 ; 15 ; 21 ; 25 ; 39 ; 45$ e 49 h, registrando-se o número de insetos em cada parte da planta.

Determinação do período de maior atividade alimentar

A atividade alimentar de B. xanthophis foi avaliada nos períodos diurno (fotofase) e noturno (escotofase), por meio da quantificação e análise da excreção de 'honeydew'. O volume excretado por unidade de tempo foi usado como medida indireta da taxa de ingestão. Neste ensaio, utilizaram-se adultos (machos e fêmeas) com idade de 5-8 dias após emergência, sendo os mesmos retirados da criação e colocados para aclimatação em plantas cítricas por $24 \mathrm{~h}$. Vinte cigarrinhas de cada sexo foram confinadas individualmente sobre a haste dos 'seedlings' (1 cigarrinha/planta), utilizando-se de uma gaiola plástica (tubo Falcon de $50 \mathrm{~mL}$ ) acoplada a um reservatório graduado na parte inferior (tubo Falcon de $15 \mathrm{~mL}$ ) (Andersen et al., 1992). As gaiolas foram posicionadas sobre a haste de modo a favorecer o 
escoamento do líquido para o recipiente graduado, permitindo a quantificação direta da excreção. $\mathrm{O}$ ensaio foi instalado no final da tarde $(18 \mathrm{~h})$ em uma sala climatizada $\left(25 \pm 2^{\circ} \mathrm{C}\right.$; fotofase de 12 h) e avaliado em períodos de $12 \mathrm{~h}$ até completar $48 \mathrm{~h}$, registrandose o volume excretado em cada período.

Baseando-se na análise química do 'honeydew' (aminoácidos ou pH) de insetos sugadores, pode-se inferir o seu local (floema ou xilema) de alimentação (Walker, 2000). Assim, para determinar o tecido de alimentação de $B$. xanthophis na fotofase e escotofase, amostras do 'honeydew' de 5 machos e 5 fêmeas por período foram submetidas à análise de $\mathrm{pH}$ em papel indicador (Universalindikator $\mathrm{pH}$, Merck) e de presença de aminoácidos em teste de reação com niidrina $(0,1 \%)$

\section{Análise dos dados}

No experimento de seleção dos locais de alimentação, utilizaram-se os testes não- paramétricos de Mann-Whitney e Behrens-Fisher para verificar a preferência dos indivíduos entre as partes superior e inferior da planta e entre partes dos ramos superiores (haste x pecíolo x limbo foliar), respectivamente. Considerou-se, como variável, o número acumulado de indivíduos registrados em todas as avaliações. Realizaram-se, também, análises de regressão para verificar a relação entre posição dos insetos na planta e tempo após a liberação na câmara de observação. Para detectar diferenças no número dos insetos presentes em certas partes da planta entre períodos de avaliação, utilizou-se o teste de Steel.

No experimento de excreção de 'honeydew', comparouse o volume médio excretado pelas cigarrinhas entre os períodos de fotofase e escotofase pelo teste não- paramétrico de Sinais de Wilcoxon, e entre machos e fêmeas pelo teste de Wilcoxon. Os testes de McNemar e de Qui-Quadrado foram usados para comparar a proporção de insetos que excretam na fotofase versus escotofase e entre machos e fêmeas, respectivamente. Todas as análises foram realizadas com o programa $\mathrm{R}$ (http://www.Rproject.org).

\section{RESULTADOS E DISCUSSÃO}

A cigarrinha B. xanthophis apresenta um local e período preferido para alimentação em mudas cítricas, comportamento este que possivelmente influencia na eficiência de transmissão de X. fastidiosa e tem implicações no manejo desse inseto vetor em pomares cítricos. No estudo de seleção do local de alimentação, B. xanthophis mostrou nítida preferência pela parte superior da muda cítrica $(\mathrm{P}<0,01)$ (Figura 1A), que é composta por ramos novos. Nesse local da planta, houve diferença significativa na ocorrência de indivíduos entre as partes do ramos $(\mathrm{P}<0,01)$, com predominância na haste (62\%), seguida pelo limbo foliar (28\%) e pecíolos (10\%) (Figura 1B).

A preferência de B. xanthophis pela parte superior da planta deve-se provalvemente à ocorrência de ramos novos. Marucci et al. (2004) observaram que duas outras cigarrinhas vetoras em citros, D. costalimai e Oncometopia facialis (Signoret), têm nítida preferência por brotações. Bi et al., (2007) verificaram que árvores cítricas jovens apresentam brotações mais vigorosas, maiores concentrações de aminoácidos na seiva do xilema e maior abundância da cigarrinha Homalodisca vitripennis (Germar) em relação a árvores mais velhas. Os níveis de aminoácidos presentes na seiva do xilema representam um dos principais fatores que influenciam na seleção de plantas hospedeiras por cigarrinhas (Brodbeck et al., 1990; Andersen et al., 2005). Assim, o fato de B. xanthophis preferir os ramos novos em mudas cítricas explicaria, pelo menos em parte, sua abundância em viveiros cítricos abertos (Roberto et al., 2000) e pomares em formação (Yamamoto et al., 2001), que geralmente apresentam maior fluxo de crescimento.

As cigarrinhas têm preferências por locais de alimentação em plantas cítricas. A espécie Acrogonia terminalis Young, por exemplo, prefere alimentar-se nas folhas novas, enquanto $D$. costalimai prefere a haste das brotações novas e Oncometopia sp. é observada na haste de brotações mais desenvolvidas, principalmente naquelas em que os ramos estão em posição vertical (Gravena et al., 1997). Em ensaios de livre escolha, realizados em laboratório, Marucci et al. (2004) observaram que a maioria dos adultos de $D$. costalimai inicialmente pousa sobre as folhas de mudas cítricas, passando posteriormente para a haste dos ramos secundários (brotações). Para $O$. facialis, não há diferença na preferência para pouso entre folhas e hastes, havendo a tendência de os insetos permanecerem nas folhas durante períodos com temperaturas mais baixas e hastes em temperatura mais quentes do dia (Marucci et al., 2004).

$\mathrm{Na}$ primeira hora após a liberação, a maioria dos indivíduos de B. xanthophis encontrava-se nas folhas (limbo foliar e pecíolos) da parte superior da planta (Figura 2). Entretanto, ao longo do tempo, observou-se uma tendência crescente na porcentagem de cigarrinhas na haste dos $\operatorname{ramos}\left(R^{2}=0,77\right)$, coincidindo com uma tendência inversa para os que estavam nas folhas $\left(\mathrm{R}^{2}=0,77\right)$ (Figura 2). A partir da terceira avaliação (21 $\mathrm{h}$ após a liberação), observou-se um aumento significativo (teste de Steel; $\mathrm{P}=0,0097$ ) na porcentagem de cigarrinhas na haste, em relação às duas primeiras avaliações do ensaio. Após $25 \mathrm{~h}$, aproximadamente $70 \%$ dos indivíduos já estavam localizados na haste (Figura 2). Esse comportamento de pouso inicial sobre as folhas e posterior movimentação para a haste dos ramos novos da planta cítrica é semelhante ao relatado para a cigarrinha $D$. costalimai (Marucci et al., 2004). Entretanto, no caso de $D$. costalimai, Marucci et al. (2004) constataram que a nervura secundária das folhas foi o local preferido de permanência (seguido da haste dos ramos) ao longo de $48 \mathrm{~h}$ de observações, em um ensaio de livre escolha, semelhante ao realizado na presente pesquisa.

Tem sido postulado que a eficiência de aquisição de $X$. fastidiosa possa estar condicionada ao local de preferência de alimentação do vetor na planta infectada, sendo maior em locais de maior concentração da bactéria (Lopes, 1996; Marucci et al., 2004). Estudando a distribuição de X. fastidiosa em citros, Queiroz-Voltan \& Paradella Filho (1999) observaram que a obstrução dos vasos do xilema diminui na direção das folhas para a haste, o que sugere maior concentração bacteriana nas folhas cítricas. O fato de D. costalimai permanecer por mais tempo sobre folhas cítricas do que $O$. facialis foi apontado por 
Marucci et al. (2004) como um dos possíveis fatores que explicariam a maior eficiência de transmissão do patógeno pela primeira espécie de cigarrinha. Entretanto, um estudo recente mostrou não haver diferença significativa entre a eficiência de transmissão de D. costalimai e B. xanthophis em citros (Marucci et al., 2008), a despeito de a segunda espécie ter preferência pela haste da planta cítrica. Assim, embora a haste apresente menor porcentagem de vasos colonizados por $X$. fastidiosa do que as folhas em citros (Queiroz-Voltan \& Paradella Filho, 1999), ela provavelmente representa um importante local de aquisição e/ ou inoculação dessa bactéria por B. xanthophis.

No experimento de excreção de 'honeydew', machos e fêmeas de $B$. xanthophis não diferiram estatisticamente entre si quanto ao volume médio excretado $(\mathrm{P}=0,76)$ e porcentagem de indivíduos que excretaram $\left(\mathrm{c}^{2}=1,11 ; \mathrm{P}=0,29\right)$. Para ambos os sexos, verificou-se maior volume de excreção $(\mathrm{P}=0,002)$ e maior porcentagem de insetos excretando durante o dia em relação à noite $\left(\mathrm{c}^{2}=10,8 ; \mathrm{P}=0,001\right)$ (Figura 3 ). Esses resultados estão coerentes com aqueles relatados por Montesino et al. (2006) para as cigarrinhas $D$. costalimai e $O$. facialis, que também apresentaram maior taxa de excreção durante o dia em ramos de laranjeiras 'Pêra' e 'Valência'.

Cigarrinhas da subfamília Cicadellinae podem sincronizar sua atividade alimentar de acordo com a flutuação química da seiva do xilema. H. coagulata, Holamodisca insolita (Walker) e Cuerna costalis (F.) alimentam-se mais ativamente sobre Catharanthus roseus (L.) durante a noite, quando a concentração de aminoácidos na seiva do xilema é 1,9 vez maior que durante o dia (Brodbeck et al., 1993). Entretanto, em Lagerstroemia indica L., essas mesmas espécies alimentam-se quase que exclusivamente durante o dia, período no qual a concentração de aminoácidos na seiva do xilema é 2,5 vezes maior em relação à noite (Brodbeck et al., 1993). Para citros, não há informações sobre variação diurna nos níveis de aminoácidos no xilema, porém, baseando-se nas maiores taxas de excreção por cigarrinhas observadas durante a fotofase no presente estudo e também por Montesino et al. (2006), é possível que a concentração de aminoácidos seja maior durante o dia. Contudo, seria necessária uma análise química detalhada da seiva do xilema de citros na fotofase e escotofase para comprovar esta hipótese.

Baseando-se nas análises de $\mathrm{pH}$ e de reação com niidrina, não houve diferenças no 'honeydew' excretado por machos e fêmeas na fotofase e escotofase. Em todas as amostras examinadas, este apresentou um $\mathrm{pH}$ inferior a 7 e não ocorreu reação com niidrina, indicando que apresenta baixa concentração ou ausência de aminoácidos. $\mathrm{O}$ pH abaixo de 7 e a falta de níveis detectáveis de aminoácidos no 'honeydew' são indicativos de ingestão no xilema, que apresenta $\mathrm{pH}$ entre 5,4-6,5 e baixa concentração de açúcares e aminoácidos (Andersen et al., 1989; Walker, 2000). Em contraste, o floema tem um pH entre 7,2-8,4 e é rico em compostos orgânicos (Walker, 2000). Assim, os dados gerados com a análise do 'honeydew' sugerem que adultos de $B$. xanthophis ingerem predominantemente conteúdos do xilema, independentemente do sexo ou período.

Pelos resultados obtidos, verificou-se que adultos de $B$. xanthophis preferem alimentar-se na haste de ramos novos em mudas cítricas, não variando de local ao longo do dia. Assim, em pomares cítricos recém-implantados ou em replantas, a inspeção e medidas de controle dessa espécie cigarrinha devem ser direcionadas neste local da planta. Como a taxa de excreção representa uma medida indireta da ingestão de seiva, os resultados do experimento de excreção de 'honeydew' apontam maior atividade alimentar durante o dia para ambos os sexos de $B$. xanthophis. Essas informações também serão úteis para aprofundar os estudos de comportamento alimentar associados à transmissão de $X$. fastidiosa.
A $\square$ Parte superior $\square$ Parte inferior

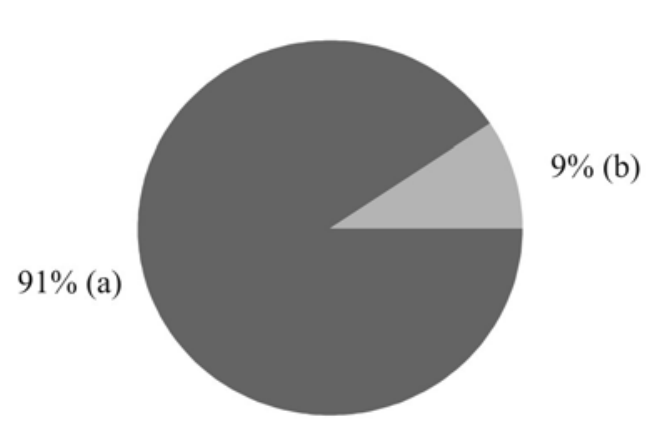

B - Haste $\quad$ Limbo foliar $\backsim$ Pecíolo

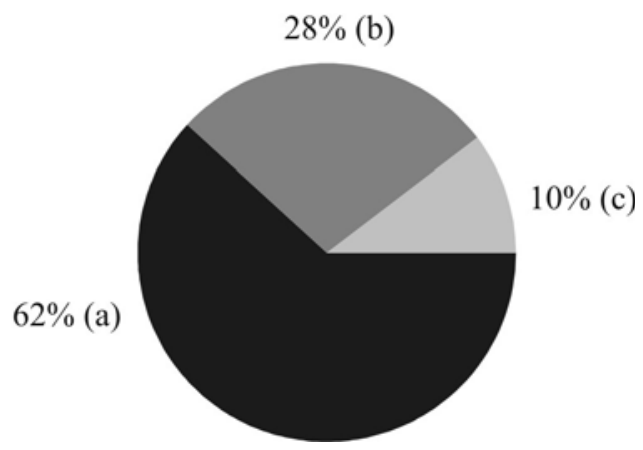

FIGURA 1- Distribuição de adultos de Bucephalogonia xanthophis em mudas de Citrus sinensis, em ensaio de livre escolha. A) Parte superior e inferior da planta; B) Haste, limbo foliar e pecíolo de ramos da parte superior da planta. Porcentagens seguidas da mesma letra, em um mesmo gráfico, não diferem entre si, pelos testes de Mann-Whitney (A) e de BehrensFisher (B) $(\mathrm{P}<0,05)$. 


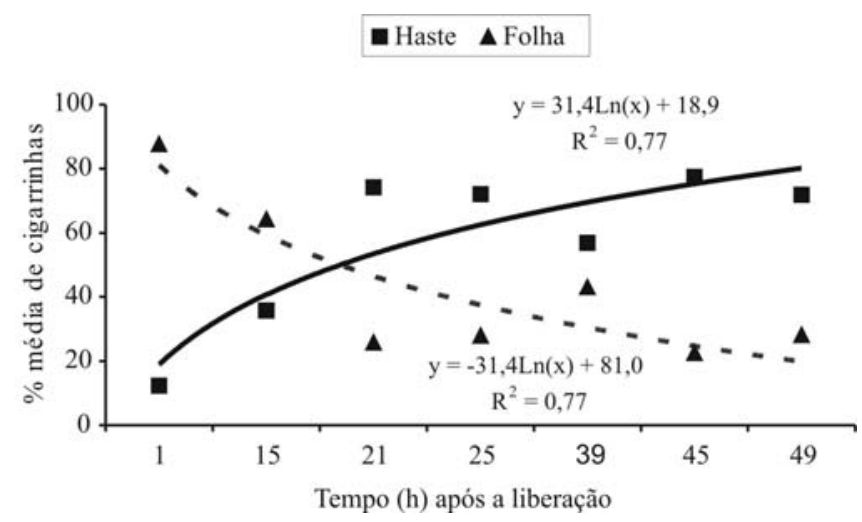

FIGURA 2- Curvas de regressão logarítmica obtidas para a relação entre a porcentagem média de adultos de Bucephalogonia xanthophis na haste ou nas folhas (limbo foliar + pecíolo) de ramos de Citrus sinensis e o tempo após a liberação dos insetos, em ensaio de livre escolha.

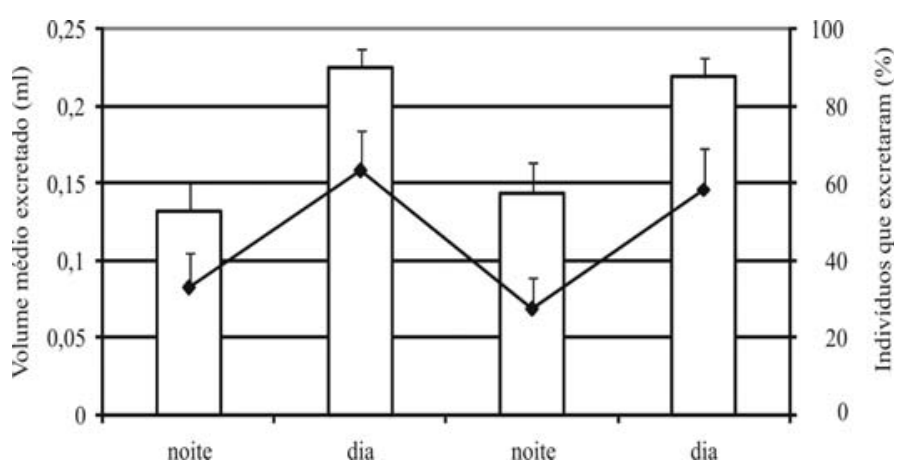

FIGURA 3- Volume médio de excreção (linha) e porcentagem de indivíduos (colunas) de Bucephalogonia xanthophis que excretaram 'honeydew' em períodos sucessivos de noite (12 h) e dia (12 h) sob confinamento em 'seedlings' de Citrus sinensis. Barras sobre a linha e colunas representam o erro-padrão das médias, as quais foram calculadas agrupando-se os dados de machos e fêmeas.

ANDERSEN, P.C.; BRODBECK, B.V.; MIZELL III, R.F. Metabolism of amino acids and organic acids and sugars extracted from the xylem fluid of 4 host plants by Homalodisca coagulata. Entomologia Experimentalis et Applicata, Dordrecht, v.50, p.149159, 1989.

1 - Hastes de ramos novos são os locais da muda cítrica onde adultos de B. xanthophis preferem alimentar-se.

2 - Durante o processo de alimentação, machos e fêmeas ingerem quantidades semelhantes de seiva do xilema.

3 - A atividade alimentar para ambos os sexos é maior durante o dia.

\section{AGRADECIMENTOS}

Ao Conselho Nacional de Desenvolvimento Científico e Tecnológico $(\mathrm{CNPq})$, pelas bolsas concedidas ao primeiro e último autores, bem como ao suporte financeiro desta pesquisa (Proc. No. 479735/2004-2). Este estudo faz parte da tese de doutorado desenvolvida pelo primeiro autor no Programa de Pós-Graduação em Entomologia, ESALQ/USP.

\section{REFERÊNCIAS}

ALMEIDA, R.P.P.; PEREIRA, E.F.; PURCEL, A.H.; LOPES, J.R.S. Multiplication and movement of a citrus strain of Xylella fastidiosa within sweet orange. Plant Disease, Saint Paul, v.85, p.382-386, 2001

ANDERSEN, P.C.; BRODBECK, B.V.; MIZELL III, R.F. Feeding by the leafhopper, Homalodisca coagulata, in relation to xylem fluid chemistry and tension. Journal of Insect Physiology, Oxford, v.38, p.611-622, 1992.
ANDERSEN, P.C.; BRODBECK, B.V.; MIZELLIII, R.F.; ODEN, S. Abundance and feeding of Homalodisca coagulata (Hemiptera: Cicadellidae) on Vitis genotypes in north Florida. Environmental Entomology, College Park, v.34, p.466-478, 2005.

BI, J.L.; CASTLE, S.J.; TOSCANO N.C. Amino acid fluctuations in young and old orange trees and their influence on glassywinged sharpshooter (Homalodisca vitripennis) population densities. Journal of Chemical Ecology, Lexington, v.33, p.16921706,2007

BRODBECK, B.V.; MIZELL III, R.F.; ANDERSEN, P.C. Physiological and behavioral adaptations of three species of leafhoppers in response to the dilute nutrient content of xylem fluid. Journal of Insect Physiology, Oxford, v.39, p.73-81, 1993.

BRODBECK, B.V.; MIZELL III, R.F.; FRENCH, W.J. Amino acids as determinants of host preference for the xylem feeding leafhopper, Homalodisca coagulata (Homoptera: Cicadellidae). Oecologia, Berlin, v.83, p.338-345, 1990.

DESCOBERTOS mais seis vetores de CVC. Revista do Fundecitrus, Araraquara, v.14, p.8-9, 1999.

GRAVENA, S.; LOPES, J.R.S.; PAIVA, P.E.B.; YAMAMOTO, P.T.; ROBERTO, S.R. Os vetores da Xylella fastidiosa. In: DONADIO, L.C.; MOREIRA, C. S. (Ed.). Clorose variegada dos citros. Bebedouro: Fundecitrus, 1997. cap. 3, p.37-53. 
KRÜGNER, R.; LOPES, M.T.V. de C.; SANTOS, J. S. et al. Transmission efficiency of Xylella fastidiosa to citrus by sharpshooters and identification of two new vector species. In: CONFERENCE OF INTERNATIONAL ORGANIZATION OF CITRUS VIROLOGISTS, 14., Campinas, 2000. Proceedings... Riverside: IOCV, 2000. p.423.

LEE, R.F.; BERETTA, M.J.G.; HARTUNG, J.H.; HOOKER, M.E.; DERRICK K.S. Citrus variegated chlorosis: confirmation of a Xylella fastidiosa as the causal agent. Summa Phytopathologica, Botucatu, v.19, p.123-125, 1993.

LOPES, J.R.S. Estudos com vetores de Xylella fastidiosa e implicações no manejo da clorose variegada dos citros. Laranja, Cordeirópolis, v.20, p.329-344, 1999.

LOPES, J.R.S. Mecanismos de transmissão de Xylella fastidiosa por cigarrinhas. Laranja, Cordeirópolis, v.17, p.79-92, 1996.

MARUCCI, R.C.; GIUSTOLIN, T.A.; MIRANDA, M.P.; MIQUELOTE, M.; ALMEIDA, R.P.P.; LOPES, J.R.S. Identification of a non-host plant of Xylella fastidiosa to rear healthy sharpshooter vectors. Scientia Agricola, Piracicaba, v.60, p.669$675,2003$.

MARUCCI, R.C.; LOPES, J.R.S.; CAVICHIOLI, R.R. Transmission efficiency of Xylella fastidiosa by sharpshooters (Hemiptera: Cicadellidae) in coffee and citrus. Journal of Economic Entomology, Lanham, 2008. no prelo

MARUCCI, R.C.; LOPES, J.R.S.; VENDRAMIM, J.D.; CORRENTE, J.E. Feeding site preference of Dilobopterus costalimai Young and Oncometopia facialis (Signoret) (Hemiptera: Cicadellidae) on citrus plants. Neotropical Entomology, Londrina, v.33, p.759-768, 2004.

MILANEZ, J.M.; PARRA, J.R.P.; CUSTODIO, I.A.; MAGRI, D.C. Feeding and survival of citrus sharpshooters (Hemiptera: Cicadellidae) on host plants. Florida Entomologist, Gainesville, v.86, p.154-157, 2003.

MIZUBUTI, E.S.G.; MATSUOKA, K.; PARIZZI, P. Associação de bactéria do tipo Xylella em laranjeiras com sintomas de clorose variegada na região da Zona da Mata de Minas Gerais. Fitopatologia Brasileira, Brasília, v.19, p.241-244, 1994.

MONTESINO, L.H.; COELHO, J.H.C.; FELIPPE, M.R.; YAMAMOTO, P.T. Ingestão de seiva do xilema de laranjeiras 'pêra' e 'valência' (Citrus sinensis (L.) Osbeck) sadias e infectadas por Xylella fastidiosa, pelas cigarrinhas vetoras Oncometopia facialis e Dilobopterus costalimai (Hemiptera: Cicadellidae). Revista Brasileira de Fruticultura, Jaboticabal, v.28, p.199-204, 2006.
PEREIRA, É.F.; LOPES, J.R.S.; TURATI, D.T.; MUNHOZ, C.; CORRENTE, J.E. Influência das condições hídricas do solo e da temperatura na sobrevivência e alimentação de Oncometopia facialis (Hemiptera: cicadellidae) em "seedlings" de citros. Arquivos do Instituto Biológico, São Paulo, v.72, p.343-351, 2005.

PURCELL, A.H. Homopteran transmission of xylem-inhabiting bacteria. In: HARRIS, K.F. (Ed.). Advances in disease vector research. New York: Springer-Verlag, 1989, p.243-266.

PURCELL, A.H.; HOPKINS, D.L. Fastidious xylem-limitedbacterial plant pathogens. Annual Review of Phytopathology, Palo Alto, v.34, p.131-151, 1996.

QUEIROZ-VOLTAN, R.B.; PARADELAFILHO, O. Caracterização de estruturas anatômicas de citros infectados com Xylella fastidiosa. Laranja, Cordeirópolis, v.20, p.55-76, 1999.

ROBERTO, S.R.; COUTINHO, A.; LIMA, J.E.O.; MIRANDA, V.S.; CARLOS, E. F. Transmissão de Xylella fastidiosa pelas cigarrinhas Dilobopterus costalimai, Acrogonia terminalis e Oncometopia facialis em citros. Fitopatologia Brasileira, Brasília, v.21, p.517-518, 1996.

ROBERTO, S.R.; PRIA JÚNIOR, W.D.; YAMAMOTO, P.T.; FELLIPE, M.R.; FREITAS, E.P. Espécies e flutuação populacional de cigarrinhas em viveiros de citros, em Gavião Peixoto (SP). Laranja, Cordeirópolis, v.21, p.65-79, 2000.

ROSSETTI, V.; GONZALEZ, M.A.; DONADIO, L.C. In: DONADIO, L.C.; MOREIRA, C.S. (Ed.). Clorose variegada dos citros. Bebedouro: Fundecitrus, 1997. cap. 1, p.1-21.

WALKER, G.P. A Beginner's Guide to Electronic Monitoring of Homopteran Probing Behavior. In: (Ed.). WALKER, G.P.; BACKUS, E.A. Principles and Applications of Electronic Monitoring and Other Techniques in the Study of Homopteran Feeding Behavior. Lanham: Thomas Say Publications in Entomology, 2000. p.14-40.

YAMAMOTO, P.T.; FELIPPE, M.R.; CAETANO, A.C.; SANCHES, A.L.; LOPES, J.R.S. First report of Fingeriana dubia Cavichioli transmitting Xylella fastidiosa to citrus. Fitopatologia Brasileira, Brasília, v.32, p.266, 2007.

YAMAMOTO, P.T.; PRIA JÚNIOR, W.D.; ROBERTO,S.R.; FELLIPE, M.R.; FREITAS, E.P. Flutuação populacional de cigarrinhas (Hemiptera: Cicadellidae) em pomar cítrico em formação. Neotropical Entomology, Londrina, v.30, p.175-177, 2001.

YAMAMOTO, P.T.; ROBERTO, S.R., PRIA JÚNIOR, W.D.; FELIPPE, M.R.; MIRANDA, V.S.; TEIXEIRA, D.C.; LOPES, J.R.S. Transmissão de Xylella fastidiosa por cigarrinhas Acrogonia virescens e Homalodisca ignorata (Hemiptera:Cicadellidae) em 